\title{
Scattering of a fluid-structure coupled wave at a flanged junction between two flexible waveguides
}

\author{
Rab Nawaz ${ }^{\text {a) }}$ and Jane B. Lawrie ${ }^{\text {b) }}$ \\ Department of Mathematics, Brunel University, Uxbridge, UB8 3PH, United Kingdom
}

(Received 10 February 2013; revised 21 July 2013; accepted 23 July 2013)

\begin{abstract}
The scattering of a fluid-structure coupled wave at a flanged junction between two flexible waveguides is investigated. The flange is assumed to be rigid on one side and soft on the other; this enables a solution to be formulated using mode-matching. It is shown that both the choice of the edge conditions imposed on the plates at the junction and the choice of incident forcing significantly affect the transmission of energy along the duct. In particular, the edge conditions crucially affect the transmission of structure-borne vibration but have little effect on fluid-borne noise. Given the singular nature of the velocity field at the flange tip, particular attention is paid to the validity of the mode-matching method. It is demonstrated that the velocity field can be accurately reconstructed by incorporating the Lanczos filter into the truncated modal expansions. The mode-matching method is thus confirmed as an viable tool for this class of problem.
\end{abstract}

(C) 2013 Acoustical Society of America. [http://dx.doi.org/10.1121/1.4817891]

PACS number(s): 43.40.Fz, 43.20.Mv, 43.40.Dx [ANN]

Pages: $1939-1949$

\section{INTRODUCTION}

Many problems involving the scattering of waves in ducts or channels are amenable to solution using analytic modematching techniques. Such methods were originally developed for canonical problems involving a governing equation such as Laplace's or Helmholtz's and in which the duct/channel boundaries were described by soft, hard, or impedance conditions. The underlying eigen-systems for this class of boundary value problem are of Sturm-Liouville type and have well defined orthogonality properties enabling the problem to be recast in terms of an infinite system of linear equations in which the unknowns are usually the scattered wave amplitudes. A key feature is that such systems are diagonally dominant and, off the diagonal, the elements decay sufficiently quickly for an accurate solution to be obtained by truncation and inversion. The standard approach fails, however, for more complicated geometries and/or ducts bounded by flexible surfaces (corresponding to high order boundary conditions) and alternative solution methods ${ }^{1,2}$ were, until recently, necessary. In recent years mode-matching methods have been devised to deal with more complicated geometries ${ }^{3-6}$ and problems involving propagation in ducts/channels with high order boundary conditions. ${ }^{7-10}$

Heating, ventilation, and air-conditioning (HVAC) ducting systems are a typical application area for mode-matching techniques. Although these methods generally neglect the effects of break-out, they do provide both physical insight into the underlying scattering processes and benchmark solutions for fully numerical approaches. HVAC noise is

\footnotetext{
${ }^{a)}$ Present Address: Department of Mathematics, Quaid-i-Azam University, Islamabad Pakistan; Permanent Address: Department of Mathematics, COMSATS Institute of Information Technology, Wah Campus, Quaid Avenue, Wah Cantt, Pakistan.

${ }^{b}$ Author to whom correspondence should be addressed. Electronic mail: jane.lawrie@brunel.ac.uk
}

generated by, for example, a fan and, at distance from the source, propagates as fluid-structure coupled waves. A typical silencer comprises a section of duct which is lined with a porous material that absorbs sound. In many cases the lining is held in place by a flange which may be curved to ease the flow of air. There is usually no change in duct height and the adjacent duct sections may be welded or riveted to the silencer.

The primary aim of this article is to explore the effects of two different sets of edge conditions, and the presence of a flange, on the propagation of a structural mode past a silencer. A simple model of the "front end" of a flexible walled silencer is considered. This comprises two semiinfinite sections of flexible-walled duct and incorporates a flange at their junction. For simplicity, instead of including a porous material, the downstream face of the flange satisfies the zero pressure condition; further, the effect of flow is neglected. A common assumption, when modeling junctions of this type, is that the duct walls are clamped at the joint. In practice, however, the duct sections are more likely to be welded together and the presence of a flange, either at the junction or in close proximity, will stiffen the joint reducing the wall displacement. Driven by these observations, this article explores the different effects that arise when the edges are (a) clamped and (b) pivoted at the junction. The former edge conditions are characterized by zero plate displacement and zero gradient, and the latter by zero displacement but continuous plate gradient and bending moment. It is worth commenting that a similar study has recently been executed for a semi-infinite ice-covered water channel; ${ }^{11}$ though that study involves different edge conditions and, of course, only reflection.

The presence of a flange ensures that the velocity field is singular in the vicinity of its tip. It is well known that many mode-matching methods fail to represent such behavior accurately. Yet mode-matching methods of the class used herein are known to conserve power ${ }^{12}$ and match pressure 
and normal velocity flux even in the presence of a corner. ${ }^{13}$ Thus, an additional aim of this paper is to investigate the accuracy of the mode-matching method in the presence of a stronger singularity. A final aim is to assess the performance of the low frequency approximation used successfully in Ref. 13.

The article is organized as follows. In Sec. II, the two-dimensional (2D) model problem is described: This incorporates a flange and/or a change in height and is thus more general than the application discussed above. The corresponding boundary value problem is stated and the singularity in the velocity field at the tip of the flange is described. The mode-matching solution is executed in Sec. III and, in Sec. IV, the low frequency (LF) approximation is derived for four geometric variations of the problem (of which, case 4 corresponds to the application discussed above). A selection of numerical results are presented in Sec. V. Section VI presents a validation of the mode-matching method. It is shown that the correct velocity field can be recovered by incorporating the Lanczos filter into the truncated modal expansions. Section VII comprises a comprehensive discussion.

\section{THE BOUNDARY VALUE PROBLEM}

The 2D waveguide under consideration comprises two distinct sections, one occupying the region $\bar{x}<0,0 \leq \bar{y} \leq \bar{a}$ and the other $\bar{x}>0,0 \leq \bar{y} \leq \bar{b}, \bar{b} \geq \bar{a}$ of a dimensional Cartesian frame of reference. The base of the waveguide, $\bar{y}=0,-\infty<\bar{x}<\infty$, is rigid while the upper surfaces (those at $\bar{y}=\bar{a}$ and $\bar{y}=\bar{b}$, respectively) comprise thin elastic plates. The two duct sections are closed by a vertical strip lying along $\bar{x}=0, \bar{d} \leq \bar{y} \leq \bar{b}, \bar{d} \leq \bar{a}$ which is assumed to be rigid on $\bar{x}=0^{-}$and to satisfy the zero pressure condition on $\bar{x}=0^{+}$. The interior region of the duct is filled with a compressible fluid of density $\rho_{a}$ and sound speed $c$. Harmonic time dependence, $e^{-i \omega \bar{t}}$, where $\omega=c k$ is the radian frequency, is assumed. It is convenient to non-dimensionalize the boundary value problem with respect to length and time scales $k^{-1}$ and $\omega^{-1}$, respectively. Thus, the non-dimensional variables are related to their dimensional counterparts through $x=k \bar{x}$ and $t=\omega \bar{t}$ etc. The non-dimensional geometry is shown in Fig. 1.

The boundary value problem is expressed in terms of the non-dimensional reduced velocity potential, and it is appropriate to define separate potentials for $x<0$ and $x>0$, thus,

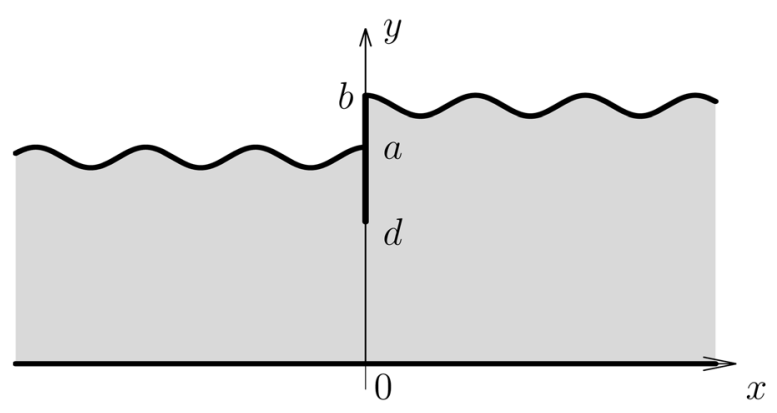

FIG. 1. The duct geometry.

$$
\phi(x, y)=\left\{\begin{array}{lll}
\phi_{1}(x, y), & x<0, & 0 \leq y \leq a \\
\phi_{2}(x, y), & x>0, & 0 \leq y \leq b .
\end{array}\right.
$$

These satisfy Helmholtz's equation with unit nondimensional wave number, that is,

$$
\left(\nabla^{2}+1\right) \phi_{j}=0, \quad j=1,2
$$

which holds throughout the fluid region and in which $\nabla^{2}$ is the Laplacian. The boundary condition at the horizontal rigid surface is

$$
\phi_{j y}=0, \quad j=1,2, \quad y=0, \quad-\infty<x<\infty,
$$

where the subscript $y$ indicates differentiation with respect to this variable. The bounding elastic plates satisfy the condition

$$
\left(\frac{\partial^{4}}{\partial x^{4}}-\mu^{4}\right) \phi_{j y}-\alpha \phi_{j}=0
$$

where for $j=1$ the condition is applied at $y=a, x<0$ and for $j=2$ it is applied at $y=b, x>0$. The non-dimensional parameters $\mu$ and $\alpha$ are the in vacuo plate wave number and a fluid loading parameter defined by

$$
\mu^{4}=\frac{12\left(1-\nu^{2}\right) c^{2} \rho_{p}}{k^{2} h^{2} E}, \quad \alpha=\frac{12\left(1-\nu^{2}\right) c^{2} \rho_{a}}{k^{3} h^{3} E},
$$

where $E$ is Young's modulus, $\rho_{p}$ is the density of the plate, $\rho_{a}$ is the density of the compressible fluid, and $\nu$ is Poisson's ratio. In this article it is assumed that the two sections of plate have identical physical properties but the method is not restricted to this case (see, for example, Ref. 12). At the matching interface, $x=0$, the fluid pressure and the normal component of velocity are continuous for $0 \leq y \leq d$, while the normal component of velocity vanishes on $x=0^{-}, d \leq y$ $\leq a$ and the fluid pressure vanishes on $x=0^{+}, d<y \leq b$. That is, at $x=0$

$$
\frac{\partial \phi_{1}}{\partial x}= \begin{cases}\frac{\partial \phi_{2}}{\partial x}, & 0 \leq y \leq d \\ 0, & d \leq y \leq a\end{cases}
$$

and

$$
\phi_{2}= \begin{cases}\phi_{1}, & 0 \leq y \leq d \\ 0, & d \leq y \leq b\end{cases}
$$

In addition to the governing equation and boundary conditions outlined above, it is necessary to apply "edge conditions" to describe how the plates are connected to the vertical rigid surface which joins the two duct sections. A comprehensive list of appropriate conditions can be found in Refs. 14-16: The two options are considered here are clamped and pivoted.

It is intended to use a mode-matching approach to solve the boundary value problem. Before the solution is presented, it is worthwhile commenting on the nature of the velocity potential as $r \rightarrow 0$ where $r=\sqrt{x^{2}+(y-d)^{2}}$ is the 
distance from the tip of the flange. For $a>d$ it is easily shown that, as $r \rightarrow 0$,

$$
\begin{aligned}
\phi(r, \theta) \sim & \beta_{0} r^{1 / 4} \sin \left[\frac{1}{4}\left(\theta-\frac{\pi}{2}\right)\right] \\
& +\beta_{1} r^{5 / 4} \sin \left[\frac{5}{4}\left(\theta-\frac{\pi}{2}\right)\right],
\end{aligned}
$$

where $\beta_{0}$ and $\beta_{1}$ are arbitrary constants and $\theta$ is the usual 2D polar angle. It is thus clear that for $a>d$ the fluid velocity is singular as $r \rightarrow 0$. It is worth noting that singular behavior similar to that displayed in (8) can be modeled by using a multi-term Galerkin approximation involving Gegenbauer polynomials. ${ }^{17-19}$ Mode-matching methods are, however, simpler to implement, known to conserve power ${ }^{12}$ and, for a velocity field with singularity of $O\left(r^{-1 / 3}\right)$ as $r \rightarrow 0$, they conserve velocity flux. ${ }^{13}$ With these points in mind, it is proposed to use this problem as a vehicle for testing the accuracy to which the mode-matching approach can model a stronger singularity.

\section{MODE-MATCHING SOLUTION}

Any incident wave propagating in the positive $x$-direction toward $x=0$ will be scattered at the discontinuity into a potentially large number of reflected and transmitted waves. The fluid velocity potentials $\phi_{j}(x, y), j=1,2$ may be expressed as eigenfunction expansions. Thus, for $x<0$,

$$
\phi_{1}(x, y)=F_{\ell} Y\left(\tau_{\ell}, y\right) e^{i \eta_{\ell} x}+\sum_{n=0}^{\infty} A_{n} Y\left(\tau_{n}, y\right) e^{-i \eta_{n} x},
$$

and for $x>0$

$$
\phi_{2}(x, y)=\sum_{n=0}^{\infty} B_{n} Y\left(\gamma_{n}, y\right) e^{i s_{n} x}
$$

Note that in Eq. (9) the forcing term comprises an arbitrary duct mode with amplitude $F_{\ell}=\sqrt{\alpha /\left(C_{\ell} \eta_{\ell}\right)}$ (chosen to ensure that the incident power is unity), and that the coefficients $A_{n}$ and $B_{n}$ are the unknown, complex amplitudes of the $n$th reflected and transmitted modes. The nondimensional modal wave numbers are $\eta_{n}=\sqrt{\tau_{n}^{2}+1}$ and $s_{n}=\sqrt{\gamma_{n}^{2}+1}$; in both cases the branch is defined such that the wave numbers are either positive real or have positive imaginary part. Further, $Y(\zeta, y)=\cosh (\zeta y)$ with $\zeta=\tau_{n}$ for $x<0$ and $\zeta=\gamma_{n}$ for $x>0, n=0,1,2, \ldots$ In expressions (9) and (10), $\tau_{n}$ and $\gamma_{n}, n=0,1,2, \ldots$ are the roots of $K(\zeta, p)=0$ where for the left hand duct $\zeta=\tau$ and $p=a$ while for the right hand duct $\zeta=\gamma$ and $p=b$. The dispersion function is

$$
K(\zeta, p)=\left[\left(\zeta^{2}+1\right)^{2}-\mu^{4}\right] \zeta \sinh (\zeta p)-\alpha \cosh (\zeta p) .
$$

The roots of $K(\zeta, p)=0$ have the following properties: (1) they occur in pairs, $\pm \zeta_{n}$; (2) there is a finite number of real roots (usually only one) but an infinite number of imaginary roots; (3) complex roots $\pm \zeta_{n}$ and $\pm \zeta_{n}^{*}$ occur for some frequency ranges. It is assumed that no root is repeated.
Positive roots, $+\zeta_{n}$, are assumed to be either positive real or have a positive imaginary part. They are ordered sequentially, real roots first and then by increasing imaginary part. Hence, $\zeta_{0}$ is always the largest real root and remaining roots are ordered accordingly. Should a complex root, say $\zeta_{n}$, lie in the upper half of the complex $\tau$-plane, then minus the complex conjugate, $-\zeta_{n}^{*}$ will also lie in the same halfplane. Such pairs are incorporated into the sequence of roots according to the magnitude of their imaginary part, and in the order $\zeta_{n}$ followed by $-\zeta_{n}^{*}$. Complex roots arise as the imaginary plate mode approaches a "cut-off" duct mode, thus a guide to the values of $k$ for which these occur is obtained by solving

$$
i \mu=\sqrt{1-\frac{n^{2} \pi^{2}}{k^{2} \bar{p}^{2}}}, \quad n=1,2,3 \ldots
$$

where $\mu$ is given in (5) and $\bar{p}$ is the dimensional height of the duct.

The generalized orthogonality relations for this class of problem are well established ${ }^{7,8,12,13}$ and are quoted here as

$$
\begin{aligned}
& \alpha \int_{0}^{a} Y\left(\tau_{m}, y\right) Y\left(\tau_{n}, y\right) d y \\
& \quad=\delta_{m n} C_{n}-\left(\tau_{m}^{2}+\tau_{n}^{2}+2\right) Y^{\prime}\left(\tau_{m}, a\right) Y^{\prime}\left(\tau_{n}, a\right),
\end{aligned}
$$

and

$$
\begin{aligned}
& \alpha \int_{0}^{b} Y\left(\gamma_{m}, y\right) Y\left(\gamma_{n}, y\right) d y \\
& \quad=\delta_{m n} D_{n}-\left(\gamma_{m}^{2}+\gamma_{n}^{2}+2\right) Y^{\prime}\left(\gamma_{m}, b\right) Y^{\prime}\left(\gamma_{n}, b\right),
\end{aligned}
$$

where, here and henceforth, the prime indicates differentiation with respect to $y$. The quantities $C_{m}$ and $D_{m}$ are given by

$$
C_{m}=\frac{\alpha a}{2}+\frac{\alpha Y\left(\tau_{m}, a\right) Y^{\prime}\left(\tau_{n}, a\right)}{2 \tau_{m}^{2}}+2 \eta_{m}^{2}\left[Y^{\prime}\left(\tau_{m}, a\right)\right]^{2},
$$

and

$$
D_{m}=\frac{\alpha b}{2}+\frac{\alpha Y\left(\gamma_{m}, b\right) Y^{\prime}\left(\gamma_{n}, b\right)}{2 \gamma_{m}^{2}}+2 s_{m}^{2}\left[Y^{\prime}\left(\gamma_{m}, b\right)\right]^{2} .
$$

The complex amplitudes, $A_{n}$ and $B_{n}$ of Eqs. (9) and (10) are, as yet undetermined and are found by appealing to continuity of pressure and normal component of velocity. On substituting (9) and (10) into (6), the velocity condition may be expressed as

$$
\begin{aligned}
& F_{\ell} \eta_{\ell} \cosh \left(\tau_{\ell} y\right)-\sum_{n=0}^{\infty} A_{n} \eta_{n} \cosh \left(\tau_{n} y\right) \\
& = \begin{cases}\sum_{n=0}^{\infty} B_{n} s_{n} \cosh \left(\gamma_{n} y\right), & 0 \leq y \leq d \\
0, & d \leq y \leq a .\end{cases}
\end{aligned}
$$

On multiplying (17) by $\alpha \cosh \left(\tau_{m} y\right)$, integrating with respect to $y, 0 \leq y \leq a$, and using (13), it is found that 


$$
\begin{aligned}
A_{m}= & F_{\ell} \delta_{\ell m}+\frac{\tau_{m} \sinh \left(\tau_{m} a\right)}{\eta_{m} C_{m}}\left[E_{1}+\left(\tau_{m}^{2}+2\right) E_{2}\right] \\
& -\frac{F_{\ell} \eta_{\ell}}{\eta_{m} C_{m}}\left(\tau_{\ell}^{2}+\tau_{m}^{2}+2\right) \tau_{\ell} \sinh \left(\tau_{\ell} a\right) \tau_{m} \sinh \left(\tau_{m} a\right) \\
& -\frac{\alpha}{\eta_{m} C_{m}} \sum_{n=0}^{\infty} B_{n} s_{n} R_{m n}
\end{aligned}
$$

where

$$
\begin{aligned}
& E_{1}=\sum_{n=0}^{\infty} A_{n} \eta_{n} \tau_{n}^{3} \sinh \left(\tau_{n} a\right), \\
& E_{2}=\sum_{n=0}^{\infty} A_{n} \eta_{n} \tau_{n} \sinh \left(\tau_{n} a\right)
\end{aligned}
$$

and

$$
R_{m n}=\int_{0}^{d} \cosh \left(\tau_{m} y\right) \cosh \left(\gamma_{n} y\right) d y,
$$

which, for $b \neq a$, simplifies to

$$
R_{m n}=\frac{\tau_{m} \sinh \left(\tau_{m} d\right) \cosh \left(\gamma_{n} d\right)-\gamma_{n} \sinh \left(\gamma_{n} d\right) \cosh \left(\tau_{m} d\right)}{\left(\tau_{m}^{2}-\gamma_{n}^{2}\right)} .
$$

To obtain a similar expression for $B_{n}, n=0,1,2, \ldots$ Eqs. (9) and (10) are substituted into (7). Thus the pressure condition may be expressed as

$$
\begin{aligned}
& \sum_{n=0}^{\infty} B_{n} \cosh \left(\gamma_{n} y\right) \\
& \quad= \begin{cases}F_{\ell} \cosh \left(\tau_{\ell} y\right)+\sum_{n=0}^{\infty} A_{n} \cosh \left(\tau_{n} y\right), & 0 \leq y \leq d \\
0, & d \leq y \leq b .\end{cases}
\end{aligned}
$$

On multiplying (23) by $\alpha \cosh \left(\gamma_{m} y\right)$, integrating with respect to $y, 0 \leq y \leq b$ and then using (14), it is found

$$
\begin{aligned}
B_{m}= & \frac{\gamma_{m} \sinh \left(\gamma_{m} b\right)}{D_{m}}\left[E_{3}+\left(\gamma_{m}^{2}+2\right) E_{4}\right]+\frac{\alpha F_{\ell}}{D_{m}} R_{\ell m} \\
& +\frac{\alpha}{D_{m}} \sum_{n=0}^{\infty} A_{n} R_{n m},
\end{aligned}
$$

where

$$
\begin{aligned}
& E_{3}=\sum_{n=0}^{\infty} B_{n} \gamma_{n}^{3} \sinh \left(\gamma_{n} b\right), \\
& E_{4}=\sum_{n=0}^{\infty} B_{n} \gamma_{n} \sinh \left(\gamma_{n} b\right) .
\end{aligned}
$$

Note that the constants $E_{1}-E_{4}$ are, as yet, unknown and are specified by enforcing the plate edge conditions.

\section{A. Clamped edges}

For the case in which both plates are clamped along the edges (i.e., at $x=0, y=a, b$ ) the appropriate edge conditions are zero displacement and zero gradient. That is,

$$
\phi_{1 y}(0, a)=0, \quad \phi_{1 y x}(0, a)=0,
$$

and

$$
\phi_{2 y}(0, b)=0, \quad \phi_{2 y x}(0, b)=0 .
$$

On comparing (27) and (28) with (20) and (26), it is clear that

$$
E_{2}=F_{\ell} \eta_{\ell} \tau_{\ell} \sinh \left(\tau_{\ell} a\right) \text { and } E_{4}=0 .
$$

In order to apply $\phi_{1 y}(0, a)=0,(18)$ is by multiplied by $\tau_{m} \sinh \left(\tau_{m} a\right)$ and, on summing over $m$, it is found that

$$
\begin{aligned}
\sum_{m=0}^{\infty} A_{m} \tau_{m} \sinh \left(\tau_{m} a\right) \\
=F_{\ell} \tau_{\ell} \sinh \left(\tau_{\ell} a\right)+E_{1} S_{1}-F_{\ell} \eta_{\ell} \tau_{\ell}^{3} \sinh \left(\tau_{\ell} a\right) S_{1} \\
\quad+\left[E_{2}-F_{\ell} \eta_{\ell} \tau_{\ell} \sinh \left(\tau_{\ell} a\right)\right] \sum_{m=0}^{\infty}\left(\tau_{m}^{2}+2\right) \frac{\tau_{m}^{2} \sinh ^{2}\left(\tau_{m} a\right)}{\eta_{m} C_{m}} \\
\quad-\alpha \sum_{m=0}^{\infty} \frac{\tau_{m} \sinh \left(\tau_{m} a\right)}{\eta_{m} C_{m}} \sum_{n=0}^{\infty} B_{n} s_{n} R_{m n} .
\end{aligned}
$$

This may be simplified using (29) and (27); it is found that

$$
\begin{aligned}
E_{1}= & -\frac{2 F_{\ell}}{S_{1}} \tau_{\ell} \sinh \left(\tau_{\ell} a\right)+F_{\ell} \eta_{\ell} \tau_{\ell}^{3} \sinh \left(\tau_{\ell} a\right) \\
& +\frac{\alpha}{S_{1}} \sum_{m=0}^{\infty} \sum_{n=0}^{\infty} \frac{B_{n} \tau_{m} \sinh \left(\tau_{m} a\right) s_{n} R_{m n}}{\eta_{m} C_{m}}
\end{aligned}
$$

where

$$
S_{1}=\sum_{m=0}^{\infty} \frac{\tau_{m}^{2} \sinh ^{2}\left(\tau_{m} a\right)}{\eta_{m} C_{m}} .
$$

On applying the same procedure for the remaining edge condition, i.e., $\phi_{2 x y}(0, b)=0$, it is found that

$$
\begin{aligned}
E_{3}= & -\frac{\alpha}{S_{2}}\left[F_{\ell} \sum_{m=0}^{\infty} \frac{s_{m} \gamma_{m} \sinh \left(\gamma_{m} b\right) R_{0 m}}{D_{m}}\right. \\
& \left.+\sum_{m=0}^{\infty} \sum_{n=0}^{\infty} \frac{A_{n} s_{m} \gamma_{m} \sinh \left(\gamma_{m} b\right) R_{n m}}{D_{m}}\right]
\end{aligned}
$$

where

$$
S_{2}=\sum_{m=0}^{\infty} \frac{s_{m} \gamma_{m}^{2} \sinh ^{2}\left(\gamma_{m} b\right)}{D_{m}}
$$

\section{B. Pivoted edges}

For the case in which the plate is pivoted along the edges, the appropriate edge conditions are zero displacement, 
continuous gradient and continuous bending moment. That is,

$$
\phi_{1 y}(0, a)=0, \quad \phi_{2 y}(0, b)=0,
$$

together with

$$
\phi_{1 y x}(0, a)=\phi_{2 y x}(0, b), \quad \phi_{1 y x x}(0, a)=\phi_{2 y x x}(0, b) .
$$

Note that, while it is possible to apply these conditions for the case $a \neq b$, they are physically realistic only when $a=b$. Application of these edge conditions gives $E_{4}=0$,

$$
\begin{aligned}
E_{1}= & \frac{1}{S_{1}}\left\{F_{\ell} \eta_{\ell} \tau_{\ell} \sinh \left(\tau_{\ell} a\right)\left[S_{3}+\left(2+\tau_{\ell}^{2}\right) S_{1}\right]\right. \\
& -2 F_{\ell} \tau_{\ell} \sinh \left(\tau_{\ell} a\right)-E_{2}\left(S_{3}+2 S_{1}\right) \\
& \left.+\alpha \sum_{m=0}^{\infty} \sum_{n=0}^{\infty} \frac{B_{n} s_{n} \tau_{m} \sinh \left(\tau_{m} a\right) R_{m n}}{\eta_{m} C_{m}}\right\}, \\
E_{2}= & F_{\ell} \eta_{\ell} \tau_{\ell} \sinh \left(\tau_{\ell} a\right)-\sum_{n=0}^{\infty} s_{n} B_{n} \gamma_{n} \sinh \left(\gamma_{n} b\right),
\end{aligned}
$$

and

$$
E_{3}=F_{\ell} \eta_{\ell}^{2} \tau_{\ell} \sinh \left(\tau_{\ell} a\right)+\sum_{n=0}^{\infty} A_{n} \eta_{n}^{2} \tau_{n} \sinh \left(\tau_{n} a\right),
$$

where

$$
S_{3}=\sum_{m=0}^{\infty} \frac{\tau_{m}^{4} \sinh ^{2}\left(\tau_{m} a\right)}{\eta_{m} C_{m}}
$$

Equations (18) and (24) together with the appropriate expressions for $E_{1}-E_{4}$ form an infinite system of linear algebraic equations from which the coefficients $A_{m}$ and $B_{m}$, $m=0,1,2, \ldots$ may be determined.

\section{A LOW FREQUENCY APPROXIMATION}

In the previous section the boundary value problem was stated and reduced, using a mode-matching procedure, to a system of equations that must be truncated and solved numerically. A low frequency (LF) approximate solution is outlined in Ref. 13, and one aim of this article is to establish the validity of this approximation. The appropriate LF expressions are thus derived here. The approximate potentials are formed from (9) and (10) simply by truncating the two eigenfunction expansions at $n=M$ and $n=N$, respectively. Thus, $M+1$ modes are taken in the reflected field and $N+1$ in the transmitted field. Only fundamental forcing is considered since the LF approximation is not expected to be valid for higher-mode forcing. Instead of using the orthogonality relations (13) and (14), the LF approximation is based on matching integral quantities such as mean pressure and velocity flux. For simplicity, the total number of modes, $M+N+2$, is chosen to be equal to the number of physical conditions to be applied at $x=0$. This means that the actual values of $M$ and $N$ depend on the relative values of $a, b$, and $d$. Continuity of mean pressure and velocity flux, however, apply in all cases.

Continuity of mean pressure is applied at $x=0$, $0 \leq y \leq d$. That is,

$$
\int_{0}^{d} \phi_{1} d y=\int_{0}^{d} \phi_{2} d y
$$

and it follows that

$$
\sum_{n=0}^{N} \frac{B_{n} \sinh \left(\gamma_{n} d\right)}{\gamma_{n}}-\sum_{n=0}^{M} \frac{A_{n} \sinh \left(\tau_{n} d\right)}{\tau_{n}}=\frac{F_{0} \sinh \left(\tau_{0} d\right)}{\tau_{0}}
$$

Continuity of velocity flux is applied at $x=0,0 \leq y \leq d$,

$$
\int_{0}^{d} \phi_{1 x} d y=\int_{0}^{d} \phi_{2 x} d y
$$

Thus,

$$
\begin{aligned}
& \sum_{n=0}^{M} \frac{A_{n} \eta_{n} \sinh \left(\tau_{n} d\right)}{\tau_{n}}+\sum_{n=0}^{N} \frac{B_{n} s_{n} \sinh \left(\gamma_{n} d\right)}{\gamma_{n}} \\
& \quad=\frac{F_{0} \eta_{\ell} \sinh \left(\tau_{0} d\right)}{\tau_{0}} .
\end{aligned}
$$

\section{A. Case 1: $a=d$ and $b>a$}

For this geometric configuration there is a change in height but no flange. Thus, in addition to (42) and (44), the average pressure at $x=0^{+}, a=d \leq y \leq b$ must be zero. That is

$$
\int_{d}^{b} \phi_{2} d y=\sum_{n=0}^{N} \frac{B_{n}\left[\sinh \left(\gamma_{n} b\right)-\sinh \left(\gamma_{n} d\right)\right]}{\gamma_{n}}=0 .
$$

Further, the plate edge conditions must be applied. For clamped edges, Eqs. (27) and (28), give

$$
\begin{aligned}
& \sum_{n=0}^{M} A_{n} \tau_{n} \sinh \left(\tau_{n} a\right)=-F_{0} \tau_{0} \sinh \left(\tau_{0} a\right), \\
& \sum_{n=0}^{M} A_{n} \eta_{n} \tau_{n} \sinh \left(\tau_{n} a\right)=F_{0} \eta_{\ell} \tau_{0} \sinh \left(\tau_{0} a\right), \\
& \sum_{n=0}^{N} B_{n} \gamma_{n} \sinh \left(\gamma_{n} b\right)=0 \\
& \sum_{n=0}^{N} B_{n} s_{n} \gamma_{n} \sinh \left(\gamma_{n} b\right)=0 .
\end{aligned}
$$

In order to accommodate all seven of the conditions at $x=0$, it is necessary to use three modes for $x<0$ and four for $x>0$. Thus, $M=2$ and $N=3$. Note that the pivoted edge conditions could be applied for $b>a$ but, as mentioned earlier, would be less physically realistic than clamped edges. 


\section{B. Case 2: $b>a>d$}

This is the most general geometry comprising a change in duct height and a flange. So in addition to Eqs. (42), (44), (45), and (46)-(49), the velocity flux at $x=0^{-}, d \leq y \leq a$ must also be zero, that is,

$$
\int_{d}^{a} \phi_{1 x} d y=0 .
$$

Hence,

$$
\begin{aligned}
& \sum_{n=0}^{M} \frac{A_{n} \eta_{n}\left[\sinh \left(\tau_{n} a\right)-\sinh \left(\tau_{n} d\right)\right]}{\tau_{n}} \\
& \quad=\frac{F_{0} \eta_{0}\left[\sinh \left(\tau_{0} a\right)-\sinh \left(\tau_{0} d\right)\right]}{\tau_{\ell}} .
\end{aligned}
$$

It follows that one more mode is needed for $x<0$, so $M=N=3$. Thus, considering only clamped edges, the coefficients for the low frequency approximation are given by Eqs. (42), (44), (45), (46)-(49) and (51).

\section{Case 3: $b=a=d$}

When $b=a=d$ there is no change in duct height and also no flange. This is the simplest duct configuration and $M=N=2$. Since $a=b$, both sets of edge conditions are physically realistic. For clamped edges the appropriate low frequency equations are Eqs. (42), (44), and (46)-(49), while for the pivoted junction they are Eqs. (42), (44), (46), and (47) together with

$$
\begin{aligned}
& \sum_{n=0}^{N} A_{n} \eta_{n} \tau_{n} \sinh \left(\tau_{n} a\right)+\sum_{n=0}^{M} B_{n} s_{n} \gamma_{n} \sinh \left(\gamma_{n} b\right) \\
& \quad=F_{0} \eta_{0} \tau_{0} \sinh \left(\tau_{0} a\right)
\end{aligned}
$$

and

$$
\begin{aligned}
& \sum_{n=0}^{N} A_{n} \eta_{n}^{2} \tau_{n} \sinh \left(\tau_{n} a\right)-\sum_{n=0}^{M} B_{n} s_{n}^{2} \gamma_{n} \sinh \left(\gamma_{n} b\right) \\
& =-F_{0} \eta_{0}^{2} \tau_{0} \sinh \left(\tau_{0} a\right) .
\end{aligned}
$$

\section{Case 4: $a=b$ and $a>d$}

In this case there is no change in duct height, but there is a flange so, as in case $2, M=N=3$. Thus, for clamped edges the appropriate low frequency equations are (42), (44), (45), (51), and (46)-(49). For pivoted edges (47) and (49) are replaced by (52) and (53).

\section{NUMERICAL RESULTS}

Given that the system of linear algebraic equations defined by (18) and (24) is suitably convergent, the solution to the physical problem can be obtained by truncating and solving the reduced system. Note that (18) and (24) hold for $m=0,1,2, \ldots$, so truncating at $m=T$ corresponds to retaining $T+1$ equations. For each graph presented in this section, the value of $T$ is stated in the figure caption. The plates were taken to be aluminum, of thickness $\bar{h}=0.0006$ $\mathrm{m}$ and of density $\rho_{p}=2700 \mathrm{~kg} \mathrm{~m}^{-3}$. In addition, the values of Young's modulus and Poisson's ratio were taken to be $E=7.2 \times 10^{10} \mathrm{Nm}^{-2}$ and $\nu=0.34$; while $c=344 \mathrm{~ms}^{-1}$ and $\rho_{a}=1.2 \mathrm{~kg} \mathrm{~m}^{-3}$, respectively.

For a duct of height $\bar{a}$, the dimensional energy flux in the positive $x$ direction per unit span in the $z$ direction is

$$
\frac{\partial \bar{E}}{\partial \bar{t}}=B\left\{\bar{w}_{\bar{x} \bar{x} \bar{x}} \bar{w}_{\bar{t}}-\bar{w}_{\bar{x} \bar{x}} \bar{w}_{\bar{x} \bar{t}}\right\}+\int_{0}^{\bar{a}} \bar{p} \frac{\partial \bar{\Phi}}{\partial \bar{x}} d \bar{y},
$$

where $B=E h^{3} /\left[12\left(1-\nu^{2}\right)\right], \bar{w}$ is the plate displacement defined by $\bar{w}_{\bar{t}}=\bar{\Phi} \bar{y}$ and $\bar{p}$ is the fluid pressure defined by $\bar{p}=-\rho_{a} \bar{\Phi}_{\bar{t}}$. Note that $\bar{\Phi}(x, y, t)$ is the total (time-dependent) dimensional velocity potential. On non-dimensionalizing this expression, averaging over one time period and expressing the result in terms of the non-dimensional time independent potentials it is found that

$$
\mathcal{E}=\frac{\partial E}{\partial t}=\Re\left\{-i \int_{0}^{a} \phi^{*} \frac{\partial \phi}{\partial x} d y-\frac{i}{\alpha}\left[\phi_{y x x x}^{*} \phi_{y}-\phi_{y x x}^{*} \phi_{y x}\right]_{y=a}\right\} .
$$

Here the integral represents the energy flux in the fluid and the remaining terms that in the plate. If $\phi_{i n c}$ is substituted into (55), where

$$
\phi_{i n c}=F_{\ell} Y\left(\tau_{\ell}, y\right) e^{i \eta_{\ell} x},
$$

it is easily shown (numerically) that, for the plate parameters considered herein and for frequencies above $50 \mathrm{~Hz}$, in excess of $99 \%$ of the energy carried by the fundamental mode $(\ell=0)$ is in the plate whereas in excess of $99 \%$ of the energy carried by the second mode $(\ell=1)$ is in the fluid. Thus, the fundamental mode is structure-borne while the second mode is fluid-borne.

On substituting the reflected field of (9) into (55), and using the orthogonality relation (13), a neat and convenient expression for the (non-dimensional) reflected energy flux across an arbitrary fictitious vertical surface in the region $x<0$ within the duct is obtained. Thus,

$$
\mathcal{E}_{1}=\frac{1}{\alpha} \sum_{j=0}^{J_{1}-1}\left|A_{j}\right|^{2} \eta_{j} C_{j}, \quad x<0,
$$

where $J_{1}$ is the number of cut-on modes in the region $x<0$. This expression, which can be found in Ref. 8 and was utilized by Warren et al. ${ }^{12}$ for a membrane bounded duct, incorporates both the fluid and the structure-borne components of the reflected energy flux. The analogous expression for the transmitted field is

$$
\mathcal{E}_{2}=\frac{1}{\alpha} \sum_{j=0}^{J_{2}-1}\left|B_{j}\right|^{2} s_{j} D_{j}, \quad x>0,
$$

where $J_{2}$ is the number of cut-on modes in the region $x>0$.

The results presented in Figs. 2-5 comprise a comparison of the reflected and transmitted components of power, 


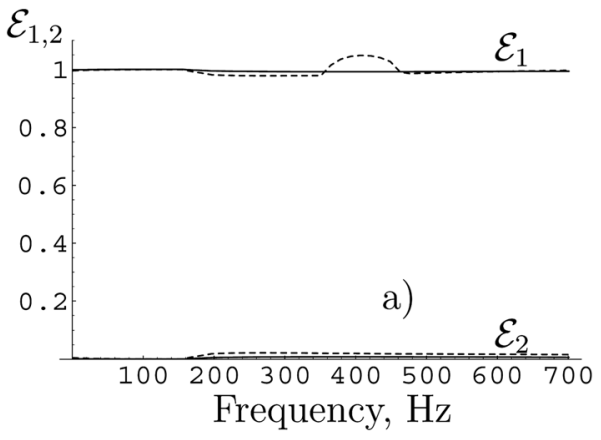

plotted against frequency, for each of the four duct configurations discussed in Sec. IV. Two different incident fields are considered: The structural-borne fundamental mode and the fluid-borne second mode. In each of Figs. 2-5 the solid curves are obtained using the mode-matching method while the dashed curves are obtained via the low frequency approximation. The latter are included only for fundamental forcing.

\section{A. Case 1: $a=d$ and $b>a$}

Figure 2 has been computed using dimensional duct heights: $\bar{a}=\bar{d}=0.06 \mathrm{~m}$ and $\bar{b}=0.085 \mathrm{~m}$. For this duct configuration only clamped edges are considered. Figure 2(a) shows the reflected and transmitted power when the forcing comprises the "structural" fundamental mode, and it is clear that the energy is almost totally reflected at the discontinuity in duct height. For the most part, the LF approximation and the mode-matching solution are in good agreement, however, the LF approximation fails for an isolated frequency interval around $400 \mathrm{~Hz}$. In this interval the dispersion relation for the left hand duct has one real root $\left(\tau_{0}\right)$, then an imaginary root $\left(\tau_{1}\right)$ which is followed by a pair of complex roots $\left(\tau_{3}\right.$ and $\left.-\tau_{3}^{*}\right)$-the presence of which is accurately predicted by (12). The complex roots are of equal physical importance, but for this duct configuration the low frequency approximation uses only three modes for the left hand duct $(M=2)$ and thus only one of the complex modes is included causing the method's failure. Once the frequency has risen to a point whereby the complex roots vanish, the previous level of accuracy is resumed. Figure 2(b) shows the reflected and transmitted power when the second mode (which cuts on at $190 \mathrm{~Hz}$ ) is incident. The incident energy is now fluid-borne and, while at cut-on the energy is almost totally reflected, the transmitted energy increases steadily with increasing frequency.

\section{B. Case 2: $b>a>d$}

In Fig. 3, $\bar{a}=0.06 \mathrm{~m}, \bar{b}=0.085 \mathrm{~m}$, and $\bar{d}=0.045 \mathrm{~m}$. As for the previous case, when the forcing comprises the fundamental mode [Fig. 3(a)] the incident energy undergoes almost total reflection at the change in duct height. While not quite over-lying, the low frequency approximation and the mode-matching solution are in good agreement for the whole of the frequency range. Figure 3(b) shows the reflected and transmitted power when the incident mode is fluid-borne (second-mode). As in case 1, the transmitted energy increases steadily with increasing frequency. Comparison of Figs. 2(b) and 3(b) suggests that the presence of the flange reduces the rate at which the transmitted energy increases with frequency.

\section{Case 3: $a=b=d$}

Case 3 corresponds to the simplest geometry in which the two duct sections are of the same height and there is no flange, thus $\bar{a}=\bar{d}=\bar{b}=0.06 \mathrm{~m}$. Eighty terms $(T=79)$ have been used to compute the graphs in Fig. 4 (this is more than necessary for fundamental forcing for which excellent results can be obtained using only 30 terms). Figures 4(a) and 4(c) correspond to fundamental forcing with clamped and pivoted edges, respectively. Both figures demonstrate a sharp inversion of the reflected and transmitted powers at the cut-on of the second duct mode. Otherwise the overall trend is almost total reflection for clamped edges and significantly more transmission for pivoted edges. For this case, the LF approach uses only three modes in each duct region $(M=N=2)$. Its failure in the region of $400 \mathrm{~Hz}$ (discussed above) is clearly visible in both Figs. 4(a) and 4(c)although for the latter accuracy is not resumed at higher frequencies. For second-mode forcing [see Figs. 4(b) and 4(d)] the vast majority of the incident energy is in the fluid and,

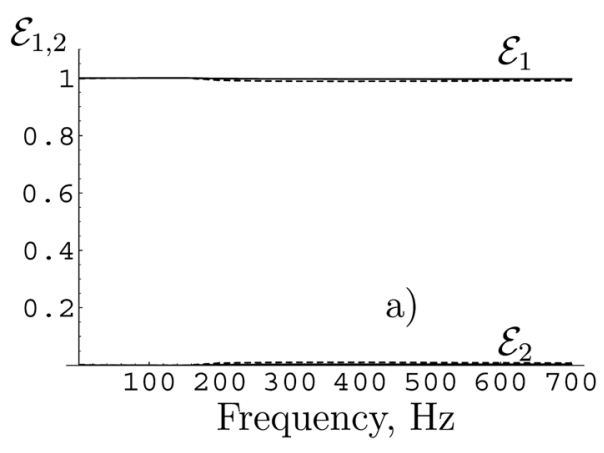

J. Acoust. Soc. Am., Vol. 134, No. 3, September 2013

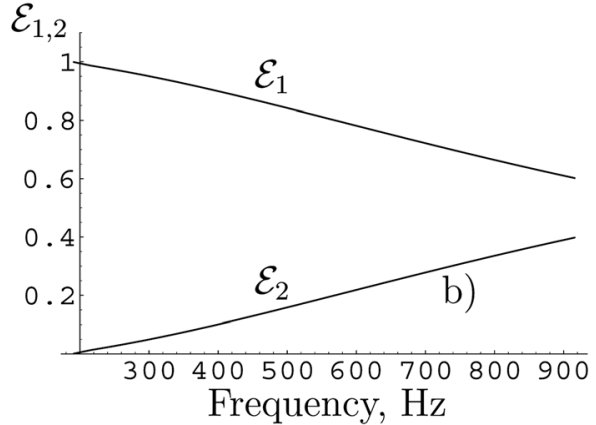

FIG. 3. Case 2, clamped edges: Forcing via (a) the fundamental mode $(T=79)$; (b) the second mode $(T=199)$. (The dashed curve is the low frequency approximation.) 

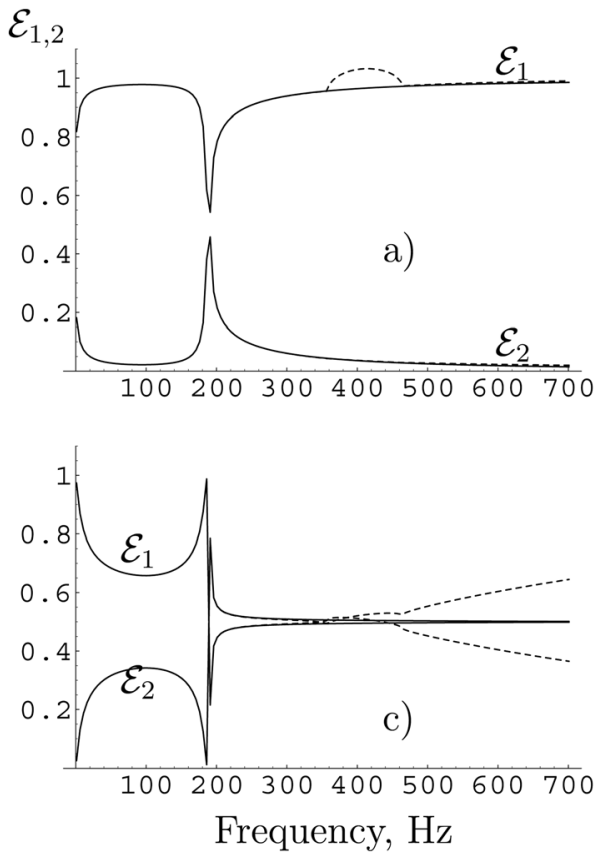

$\mathcal{E}_{1,2}$
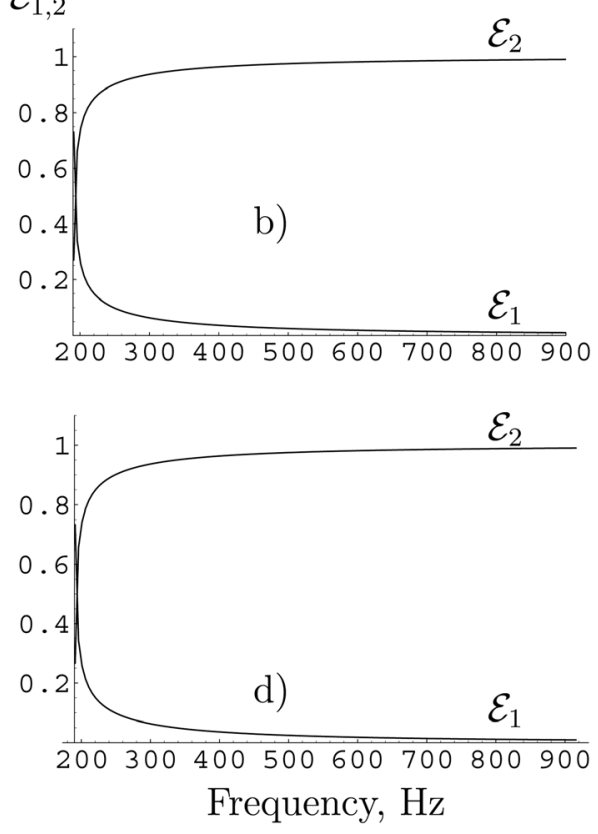

FIG. 4. Case 3: (a) Clamped edges, fundamental forcing; (b) clamped edges, second-mode forcing; (c) pivoted edges, fundamental forcing; (d) pivoted edges, second-mode forcing. In each case, $T=79$. for frequencies above $300 \mathrm{~Hz}$, very little is reflected at the duct junction. Further, it is clear that the plate edge conditions have little or no effect on the reflection/transmission of energy.

\section{Case 4: $a=b$ and $a>d$}

In Fig. 5 the dimensional height of both duct sections is $\bar{a}=\bar{b}=0.06 \mathrm{~m}$ while $\bar{d}=0.045 \mathrm{~m}$. Figures $5(\mathrm{a})$ and $5(\mathrm{c})$ compare the clamped and pivoted edge conditions for fundamental forcing. As above, when both plates are clamped there is almost total reflection of the incident power with only a small fraction being transmitted into the duct lying in $x>0$. In contrast Fig. 5(c) shows that when the plates are pivoted the reflected and transmitted powers are almost equal for frequencies above $400 \mathrm{~Hz}$. Since this geometric configuration includes a flange (so that $M=N=3$ ), the loss of accuracy in the LF approximation remarked upon above does not occur around $400 \mathrm{~Hz}$. There is, however, a small but discernable discrepancy between the LF approach and the mode-matching approach in the region of $200 \mathrm{~Hz}$. This coincides with the cut-on frequency for the second duct mode. Figures 5(b) and 5(d) compare the clamped and pivoted edge conditions for second-mode forcing. As in case 3 [see Figs. 4(b) and 4(d)], the vast majority of the incident energy is in the fluid. The effect of the flange is clear in that the transmitted energy increases with frequency much more slowly than in case 3 . The plate edge conditions again have no apparent effect on the reflection/transmission of energy.

\section{VALIDATION OF THE METHOD}

The results presented in Sec. V focus on the reflected and transmitted energies for two different incident waves
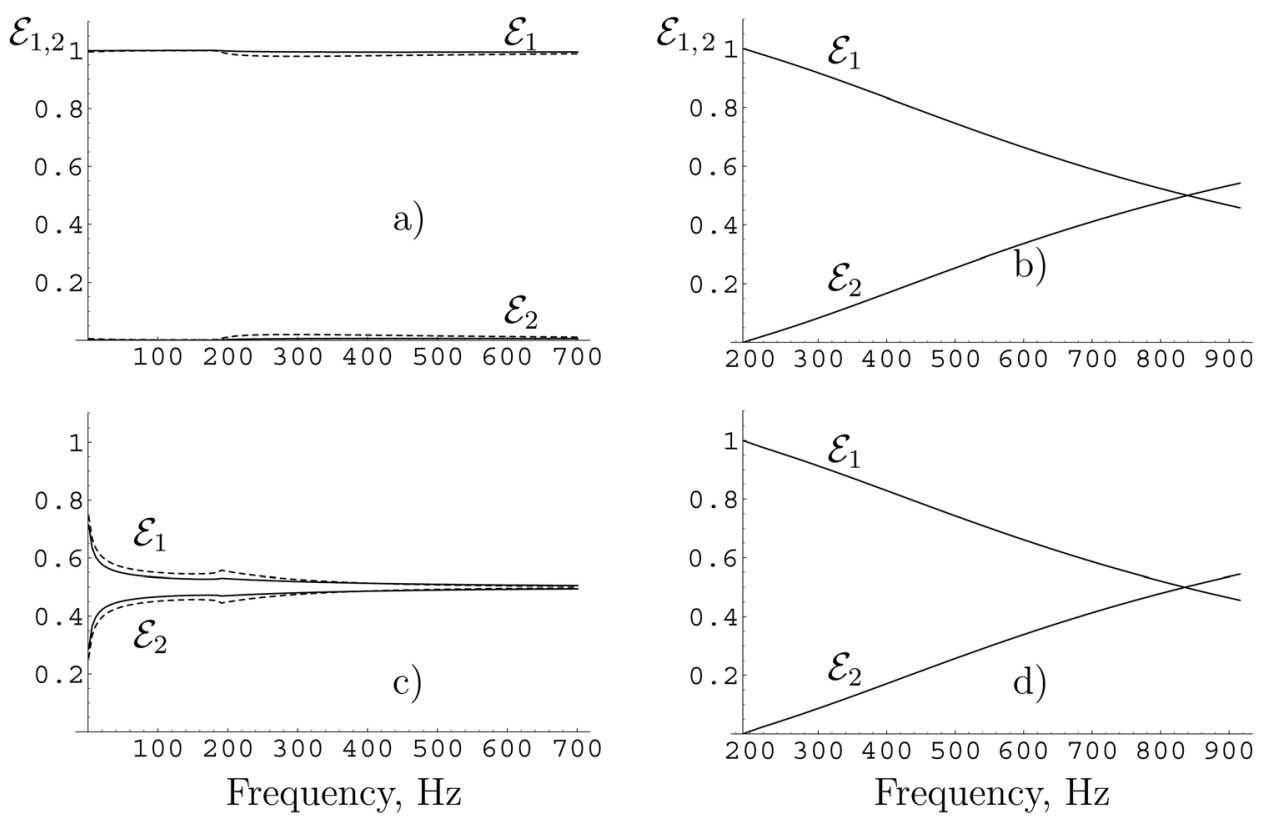

FIG. 5. Case 4: (a) Clamped edges, fundamental forcing; (b) clamped edges, second-mode forcing; (c) pivoted edges, fundamental forcing; (d) pivoted edges, second-mode forcing. In each case, $T=199$. 


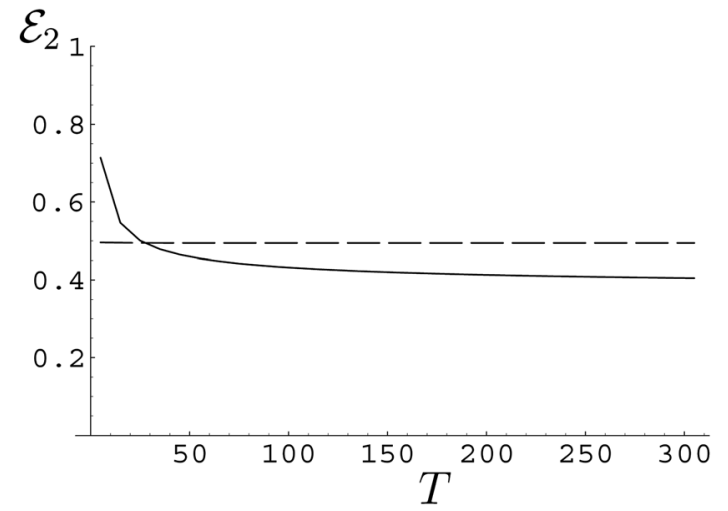

FIG. 6. Case 4: Transmitted energy against truncation parameter, $T$, for pivoted edges at $700 \mathrm{~Hz}$. The dashed line corresponds to fundamental forcing and the solid line to second-mode forcing.

and two different sets of edge conditions. Bearing in mind the nature of the singularity in the velocity field, it is essential to validate the mode-matching method. This can be done by confirming, first, that sufficient terms have been included in the truncation of Eqs. (18) and (24) to ensure that the modal coefficients have converged adequately and, second, that the coefficients contain the correct information to enable the matching conditions, (6) and (7), to be reconstructed. The next three figures examine these issues for case 4 [the singularity structure for which is given in (8)] with pivoted edges.

Figure 6 shows the transmitted energy against the truncation parameter at $700 \mathrm{~Hz}$. [Recall that (18) and (24) hold for $m=0,1,2, \ldots$, so truncating at $m=T$ corresponds to retaining $T+1$ equations.] Clearly, the system converges more slowly when the second-mode is incident and attention is henceforth restricted to this forcing with $T=199$. Figure 7(a) shows the real parts of the non-dimensional pressures at the matching interface, that is $\Re\left\{\phi_{1}(0, y)\right\}$ and $\Re\left\{\phi_{2}(0, y)\right\}$, $0 \leq y \leq a$, at $700 \mathrm{~Hz}$. In the fluid region, $0 \leq y \leq d$, there is good agreement between the two curves and, furthermore, $\Re\left\{\phi_{2}(0, y)\right\} \approx 0$ for $d \leq y \leq a$ indicating that the real part of pressure does satisfy condition (7) (the same level of accuracy is observed in the imaginary parts). Figure 7(b) shows the imaginary parts of the normal components of velocity, $\Im\left\{\phi_{1 x}(0, y)\right\}$ and $\Im\left\{\phi_{2 x}(0, y)\right\}, 0 \leq y \leq a$. All useful information is obscured by Gibb's phenomenon.

Gibb's phenomenon is caused by the abrupt truncation of a poorly convergent series. Much research has been done into the resolution of this phenomenon and, in particular, how to accurately reconstruct a function given its Fourier coefficients. $^{20-22}$ The Lanczos filter provides a simple and effective means of doing this. For the sake of completeness, the pertinent properties of the filter are briefly summarized.

The results presented in Fig. 7 were obtained by truncating the modal expansions and plotting partial sums of the form

$$
f_{T}(y)=\sum_{n=0}^{T} A_{n} \cosh \left(\tau_{n} y\right), \quad-a \leq y \leq a,
$$

where $T+1$ terms are have been retained. Consider instead the quantity $f_{T}^{\sigma}(y)$ where

$$
f_{T}^{\sigma}(y)=\frac{T}{2 a} \int_{-a / T}^{a / T} f_{T}(y+v) d v .
$$

This is the convolution of the partial sum $f_{T}(y)$ with the function

$$
S(v)= \begin{cases}T /(2 a), & |v|<a / T \\ 0, & |v|>a / T .\end{cases}
$$

Clearly as $T \rightarrow \infty, S(v) \rightarrow \delta(v)$ (Dirac's delta function) and $f_{T}^{\sigma}(y) \rightarrow f_{T}(y) \rightarrow f(y)$. By direct integration of $(60)$, it is found that

$$
f_{T}^{\sigma}(y)=\sum_{n=0}^{T} A_{n} \sigma_{n} \cosh \left(\tau_{n} y\right)
$$

where

$$
\sigma_{n}=\frac{\sinh \left(\tau_{n} a / T\right)}{\tau_{n} a / T} .
$$

The quantity $\sigma_{n}$ is the Lanczos filter (also known as a sigma factor). Its inclusion in the partial sum aids convergence and smoothes out Gibb's phenomenon thus providing a more accurate approximation to $f(y)$ everywhere on $(-a, a)$ except at the singularity. On noting that, for the parameters and frequency ranges considered herein,

$$
\tau_{n+2} \sim \frac{i n \pi}{a}, \quad n>1,
$$

it is clear that $\sigma_{0} \rightarrow 1$ while $\sigma_{n} \rightarrow 0$, as $n \rightarrow T \rightarrow \infty$. Thus, the filter does not affect each term of the partial sum in the same way. The first few terms are unaffected but as $n$ increases the sigma factor successively reduces the significance of each term. It is also worth commenting this filter is generally used

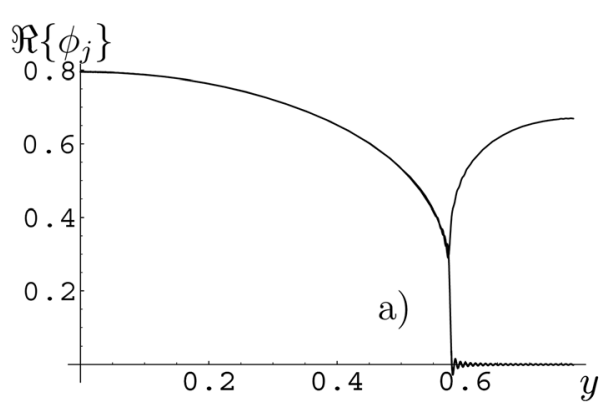

FIG. 7. Case 4, pivoted edges and second-mode forcing at $700 \mathrm{~Hz}$ : (a) The real parts of $\phi_{j}(0, y)$; (b) the imaginary parts of $\phi_{j x}(0, y), j=1,2$. 


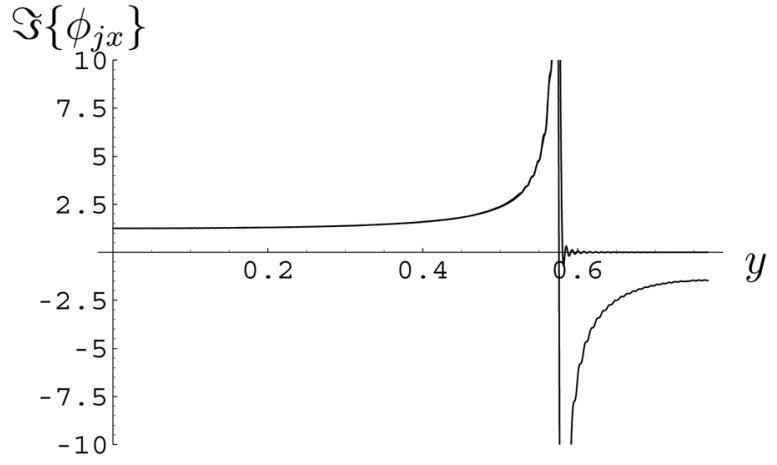

FIG. 8. Case 4, pivoted edges and second-mode forcing: The imaginary parts of $\phi_{j x}(0, y), j=1,2$ computed using the Lanczos filter.

with standard Fourier series, however, its effectiveness for this class of modal expansion is assured by (64). The reader is referred to Ref. 21 and Ref. 22 for further details of this and similar filters, including error bounds for $\left|f_{T}^{\sigma}(y)-f(y)\right|$.

Figure 8 shows the imaginary parts of $\phi_{1 x}(0, y)$ and $\phi_{2 x}(0, y), 0 \leq y \leq a$, computed using the filter, for case 4 (second-mode forcing) with pivoted edge conditions at $700 \mathrm{~Hz}$ and with $T=199$. This figure should be compared with Fig. 7(b). While the filter has not completely removed the oscillations, they are significantly smoothed. Further, the velocities are in excellent agreement in the fluid region and $\phi_{1 x}(0, y) \approx 0$ for $\mathrm{d} \leq y \leq a$ as required. The curves could be further smoothed by either increasing $T$ or repeated applications of the filter, but this is not considered necessary for the purposes of this article. It is clear that matching condition (6) has been reconstructed using the modal coefficients obtained by truncating Eqs. (18) and (24). This validates the method and the numerical results presented in Sec. V.

\section{DISCUSSION}

The scattering of fluid-coupled structural waves by a geometric discontinuity, comprising a flange and/or a change in height, in a 2D duct has been studied. The model problem was solved using an established mode-matching method and a range of numerical results have been presented for two sets of edge conditions and two different incident modes. A number of interesting physical features have been illustrated.

When forcing is provided via the structure-borne fundamental mode, the transmission and reflection of energy is significantly affected by the choice of edge conditions. If the plates are clamped at $x=0$, the vast majority of the incident energy is simply reflected, and very little is transmitted into the region $x>0$. In contrast, when $a=b$ and the plates are pivoted at $x=0$ the proportion of energy transmitted is much higher-approaching 50\% of the total energy for frequencies above $400 \mathrm{~Hz}$. These observations hold with or without a flange and are consistent with the energy being structure borne. On the other hand, for second-mode forcing, most of the energy is transported in the fluid and the edge conditions have no apparent effect on the reflected and transmitted fields. These findings have implications for the design of ducting systems in which adjacent duct sections may be riveted or welded together. Mathematically, a welded junction is characterized by continuity of plate displacement, gradient, bending moment, and force which are ideal conditions for a structure-borne mode to propagate with limited reflection.

The results obtained using mode-matching have been compared with those for a low frequency (LF) approximation. It has been shown that the latter method does surprising well for the (small) ducts and moderate frequency ranges used herein. The LF approach fails in situations where a pair of complex roots cannot both be included in the approximation (see Figs. 2 and 4) and, of course, with increasing frequency. Further, the LF method cannot impose the correct continuity conditions at the matching interface. Instead, it matches average pressure and velocity flux and thus the approach should never be used for situations in which details of the actual pressure or velocity are required. It is a potentially useful tool, however, for an initial investigation and/or for verifying numerical/analytic results in the low frequency regime.

The choice of model problem facilitated an investigation into the accuracy of the mode-matching solution given a singular velocity field. This method relies on the analytic properties of the eigenfunctions corresponding to acoustic propagation in a uniform duct. It is well known that in the absence of a singularity the pressure and normal velocity are matched to a high level of accuracy, but mode-matching methods of this type are not usually expected to model singularities accurately. The results presented herein, however, show that the method copes well with the discontinuous pressure condition caused by the presence of a flange [see Fig. 7(a)] and, more surprisingly, that the correct velocity field can be recovered with the aid of the Lanczos filter (see Fig. 8). A disadvantage of the method is the large number of terms required to ensure accuracy.

Finally, it is remarked that the model problem solved herein features a "soft-backed" flange, that is, one which is rigid on one side $\left(x=0^{-}\right)$and soft on the other $\left(x=0^{+}\right)$. This configuration is amenable to the analytic solution method presented herein. The case of a totally rigid flange is a more challenging variation of the problem. (See, for example, Ref. 23 in which the main problem is recast in terms of a duct with a flange but in which the walls are not flexible.) Nevertheless, given the surprisingly good results presented in Sec. V, the LF method would seem a useful tool for a preliminary investigation in the low frequency regime.

\section{ACKNOWLEDGMENTS}

R.N. gratefully acknowledges the financial support of the Higher Education Commission, Pakistan.

${ }^{1}$ I. D. Abrahams, "Scattering of sound by finite elastic surfaces bounding ducts or cavities near resonance," Q. J. Mech. Appl. Math. 35(1), 91-101 (1982).

${ }^{2}$ L. Huang, "Modal analysis of a drumlike silencer," J. Acoust. Soc. Am. 112, 2014-2025 (2002).

${ }^{3}$ R. Kirby, "Modeling sound propagation in acoustic waveguides using a hybrid numerical method," J. Acoust. Soc. Am. 124(4), 1930-1940 (2008).

${ }^{4} \mathrm{R}$. Kirby, "A comparison between analytic and numerical methods for modelling automotive dissipative silencers with mean flow," J. Sound Vib. 325, 565-582 (2009). 
${ }^{5}$ W. Bi, V. Pagneux, D. Lafarge, and Y. Aurégan, “An improved multimodal method for sound propagation in nonuniform lined ducts," J. Acoust. Soc. Am. 122, 280-290 (2007).

${ }^{6} \mathrm{P}$. A. Martin, "The horn-feed problem: sound waves in a tube joined to a cone, and related problems," J. Eng. Math 71, 291-304 (2011).

${ }^{7}$ J. B. Lawrie and I. D. Abrahams, "An orthogonality condition for a class of problems with high order boundary conditions; applications in sound/ structure interaction,” Q. J. Mech. Appl. Math. 52, 161-181 (1999).

${ }^{8} \mathrm{~J}$. B. Lawrie, "Comments on a class of orthogonality relations relevant to fluid-structure interaction," Meccanica 47(3), 783-788 (2012).

${ }^{9}$ Mahmood-Ul-Hassan, M., H. Meylan, and M. A. Peter, "Water-wave scattering by submerged elastic plates," Q. J. Mech. Appl. Math. 62, 321-344 (2009).

${ }^{10}$ S. C. Mohapatra, D. Karmakar, and T. Sahoo, "On capillary gravity-wave motion in two-layer fluids," J. Eng. Math. 71(3), 253-277 (2011).

${ }^{11} \mathrm{~J}$. Bhattacharjee and C. Guedes Soares, "Flexural gravity wave over a floating ice sheet near a vertical wall," J. Eng. Math. 75(1), 29-48 (2012).

${ }^{12}$ D. P. Warren, J. B. Lawrie, and I. M. Mohamed, "Acoustic scattering in waveguides that are discontinuous in geometry and material property," Wave Motion 36, 119-142 (2002).

${ }^{13}$ J. B. Lawrie and I. M. M. Guled, "On tuning a reactive silencer by varying the position of an internal membrane," J. Acoust. Soc. Am. 120, 780-790 (2006).
${ }^{14}$ P. R. Brazier-Smith, "The acoustic properties of two co-planar half-plane plates,” Proc. R. Soc. A. 409, 115-139 (1987).

${ }^{15}$ A. N. Norris and G. R. Wickham, "Acoustic diffraction from the junction of two flat plates,” Proc. R. Soc. A. 451, 631-655 (1995).

${ }^{16}$ J. B. Lawrie and I. D. Abrahams, "Scattering of fluid loaded elastic plate waves at the vertex of a wedge of arbitrary angle, I: Analytic solution," IMA J. Appl. Math. 59, 1-23 (1997).

${ }^{17}$ D. V. Evans and M. Fernyhough, "Edge waves along periodic coastlines, Part 2," J. Fluid Mech. 297, 307-325 (1995).

${ }^{18}$ M. Kanoria, D. P. Dolai, and B. N. Mandal, "Water-wave scattering by thick vertical barriers,” J. Eng. Math. 35, 361-384 (1999).

${ }^{19}$ D. Homentcovschi and R. N. Miles, "A re-expansion method for determining the acoustical impedance and the scattering matrix for the waveguide discontinuity problem," J. Acoust. Soc. Am. 128(2), 628-638 (2010).

${ }^{20}$ C. E. Duchon, "Lanczos filtering in one and two dimensions," J. Appl. Meteorol. 18, 1016-1022 (1979).

${ }^{21}$ H. Vandeven, "Family of spectral filters for discontinuous problems," J. Sci. Comput. 8, 159-192 (1991).

${ }^{22}$ D. Gottlieb and C.-W. Shu, "On the Gibb's phenomenon and its resolution," SIAM Rev. 39(4), 644-668 (1997).

${ }^{23}$ B. Erbas and I. D. Abrahams, "Scattering of sound waves by an infinite grating composed of rigid plates," Wave Motion 44(4), 282-303 (2007). 